Proc. Indian Acad. Sci. (Chem. Sci.), Vol. 89, Number 2, April 1980, pp. 209-217. (C) Printed in India.

\title{
Effect of adsorption of vapours on the electrical conductivity of some polyene semiconductors: adsorption and desorption kinetics
}

\author{
ALPANA GHOSH, BISWANATH MALLIK and T N MISRA \\ Optics Department, Indian Association for the Cultivation of Science, Jadavpur, \\ Calcutta 700 032, India \\ MS received 30 October 1979; revised 16 January 1980

\begin{abstract}
The change in semiconductive properties of $\beta$-apo- 8 '-carotenal, astacene and methyl bixin on adsorption of various vapours on the crystallite surfaces has been studied at a constant sample temperature. The adsorption of vapours enhances the semiconductivity of the polyenes appreciably. This enhancement depends on the chemical nature and also on the pressure of the adsorbed vapour. The adsorption and desorption kinetics follow the modified Roginsky-Zeldovich relation. A two stage adsorption process, the first stage of which gives a Lennard-Jones potential energy curve and is followed by a rate-determining transition over a potential energy barrier to the second stage of adsorption forming weakly bound complexes between the vapour molecules and the polyene crystallites, can explain satisfactorily the experimentally observed kinetic data.
\end{abstract}

Keywords. Organic semiconductors ; vapour adsorption effect ; desorption kinetics; adsorption kinetics; electrical conductivity; polyene semiconductors.

\section{Introduction}

The effect of adsorption of various vapours and gases on the semiconductive properties of polyenes are of considerable interest as the change in conductivity of these compounds on vapour or gas adsorption could be involved in the primary mechanism of olfactory transduction process (Rosenberg et al 1968; Misra et al 1968). In an earlier paper (Mallik et al 1979a) we have reported that the adsorption of vapours of benzene, toluene, ethyl acetate, heptane, ethanol and methanol on vitamin A (alcohol and acetate) enhances the semiconduction current and decreases the semiconduction activation energy appreciably. The adsorption kinetics were observed (Mallik et al 1979b) to follow the modiffed RoginskyZeldovich equation which assumes that the rate of adsorption possesses an activation energy which increases linearly with the amount of adsorbed vapour. Recently (Mallik et al 1980) we have observed that the adsorption of these vapours on the polycrystals of some other polyenes, namely $\beta$-apo- $8^{\prime}$-carotenal, astacene and methyl bixin also enhances the specific conductivities of the compounds but unlike that in vitamin $A$, increases the activation energies. It was, therefore, 
considered worthwhile to examine if the similar adsorption kinetics are follow in these polyenes also. In this paper we examine the adsorption and desorption kinetics, and estimate the activation energies of adsorption and desorption from the kinetic data analysis and discuss a possible model for the adsorption process.

\section{Experimental}

The polyene semiconductors studied are $\beta$-apo- $\beta^{\prime}$-carotenal, astacene and methyl bixin. These high quality compounds were obtained from Hoffman and LaRoche Co. Limited, Switzerland, and have been used without further purification. The reagent chemicals, toluene, benzene, ethyl acetate, $n$-heptane, ethanol ard methanol used in this experiment were of spectrograde quality (BDH and E Merck). The experimental arrangement is similar to that reported in the earlier communication (Mallik et al 1979b).

\section{Results}

\subsection{Semiconduction of the polyenes and the effect of adsorption of vapours}

The effects of adsorption of vapours were studied in the usual manner by applying the sandwich cell technique. The sandwich cell was temperature-cycled and in addition dry nitrogen gas was allowed to pass through the chamber to desorb any vapour or gas adsorbed by the sample prior to the experiment. The cell was then kept at room temperature $\left(25^{\circ} \mathrm{C}\right)$ and the carrier gas, dry nitrogen, was passed through the reagent liquid which was kept at a constant temperature to maintain a desired vapour pressure. As the powder sample adsorbs the vapour from the chamber atmosphere, the current increases and finally attains a saturation value. The current enhancement was by several orders of magnitude in some cases. The result of such a measurement for ethyl acetate vapour adsorption in $\beta$-apo- $8^{\prime}$-carotenal is shown in figure 1. When the chamber is flushed with dry nitrogen gas, the vapour is desorbed from the crystallite surfaces and the current comes back to its initial value. This is shown in curve (b) of ftgure 1. The arrow indicates the time when desorption starts. Such adsorption and desorption kinetic curves were obtained with other vapours. The other polyenes also show similar adsorption and desorption curves. The maximal value of current reached under particular experimental conditions, depends on the vapour pressure of the reagent liquid at its temparatire and also on the temperature of the sample cell; the time to reach this value depends also on the flow rate. To test the sensitivity of a particular polyene semiconductor for adsorption of different vapours, saturation current values were noted after adsorption of various vapours at a fixed partial vapour pressure with a constant flow rate and a constant sample cell temperature $\left(18 \cdot 5^{\circ} \mathrm{C}\right)$. The sensitivity as measured by $\sigma_{A} / \sigma_{V}$ values where $\sigma_{A}$ is the specific conductivity after vapour adsorption and $\sigma_{V}$ is that of before adsorption. The results are summarised in table 1. Apparently, the sensitivity depends on the chemical nature of the adsorbed molecules. 


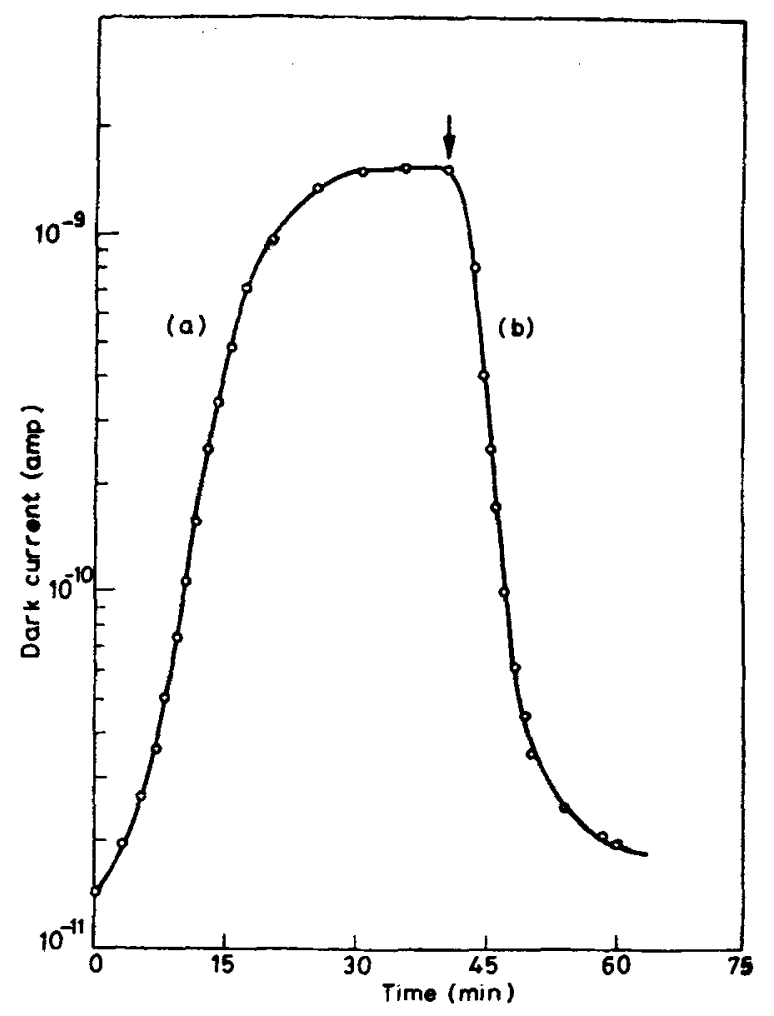

Figure 1. Change in dark current in a $\beta$-apo- $8^{\prime}$-carotenal powder cell kept at $25^{\circ} \mathrm{C}$ with (a) adsorption and (b) desorption of ethyl acetate vapour at $59.5 \mathrm{~mm}$ pressure.

\subsection{Semiconduction as a function of vapour pressure}

We have studied the magnitude of the current increase at a constant sample cell temperature $\left(25^{\circ} \mathrm{C}\right)$ as a function of the partial pressure of the vapours in the chamber. To change this partial pressure, the temperature of the reagent liquid through which dry nitrogen gas was passed and fed into the conductivity chamber was varied. The steady-state current was noted for different vapour pressures. The kinetics for the dark current enhancement for different partial pressures of ethyl acetate ambient vapour for $\beta$-apo- $8^{\prime}$-carotenal is shown in figure 2 . The time required for reaching the saturation current increases with the increasing vapour pressure as at saturation more vapours get adsorbed at higher pressure (Eley and Leslie 1964). Similar results were obtained with other vapours. The other polyenes also show similar behaviour.

\section{Discussion and conclusions}

\subsection{Dependence of the conductivity on vapour pressure}

We have shown (Mallik et al 1979b) in vitamin A (alcohol and acetate) that the relation between the specific conductivity $\sigma_{A}\left(m_{0}\right)$ at equilibrium and the pressure (p) of ambient vapour is given by

$$
\sigma_{A}\left(m_{0}\right)=\sigma_{V} \exp \left(\alpha Q_{\ominus} p\right),
$$

P.(A) -15 
Table 1. Rise in the dark current in the powder cells of some polyenes at $18 \cdot 5^{\circ} \mathrm{C}$ due to adsorption of various vapours at the same pressure $(p)$ for a particular polyene.

\begin{tabular}{|c|c|c|c|}
\hline \multirow{2}{*}{ Vapour adsorbed } & \multicolumn{3}{|c|}{$\sigma_{A} / \sigma_{V}$} \\
\hline & $\begin{array}{c}\beta \text {-apo- } 8^{\prime} \text {-carotenal } \\
p=42 \mathrm{~mm}\end{array}$ & $\begin{array}{c}\text { Astacene } \\
p=50 \mathrm{~mm}\end{array}$ & $\begin{array}{l}\text { Methyl bixin } \\
p=60 \mathrm{~mm}\end{array}$ \\
\hline
\end{tabular}

$\begin{array}{llll}\text { Toluene } & 7.3 & 1.8 & 1.5 \\ \text { Benzene } & 4.2 & 2.5 & 3.3 \\ \text { Ethyl acetate } & 5.0 \times 10^{2} & 1.5 \times 10^{2} & 1.0 \times 10^{1} \\ \text { n-Heptane } & 4.4 & . & . \\ \text { Ethanol } & 5.0 \times 10^{3} & 4.0 \times 10^{3} & 1.0 \times 10^{2} \\ \text { Methanol } & 6.5 \times 10^{2} & 5.0 \times 10^{2} & 3.3 \times 10^{1}\end{array}$

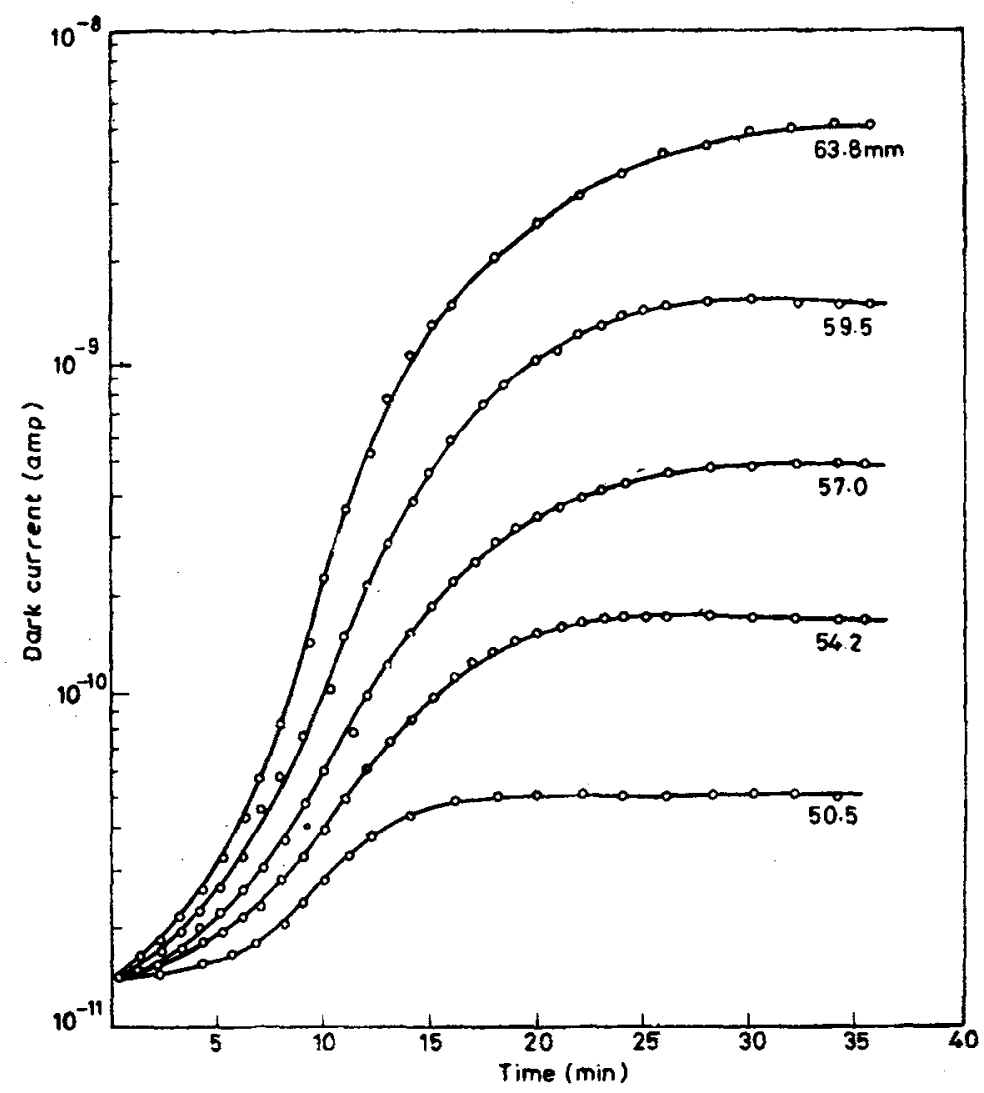

Fotre 2. Change in dark current in a $\beta$-apo- $8^{\prime}$-carotenal powder cell kept at $25^{\circ} \mathrm{C}$ after adsorption of ethyl acetate vapour at different pressures. 
where $m_{0}$ is the amount of vapour adsorbed at equilibrium, $\alpha$ and $Q_{0}$ are constants. According to equation (1), a plot of $\log \sigma_{A}\left(m_{0}\right)$ versus the vapour pressure $(p)$ at equilibrium is expected to be linear. In figure 3 we show such plots of logarithm of the saturation currents versus vapour pressure $(p)$ at equilibrium for ethyl acetate adsorption on various polyene semiconductors. Fairly good straight lines are obtained. The slope of these curves $\left(a Q_{0}\right)$ is a measure of the strength of interaction between the vapour molecules and the semiconductors. The values of $a Q_{0}$ as obtained from the slopes of the lines in figure 3 are $0.354,0.089$ and $0: 078 \mathrm{~mm}^{-1}$ for $\beta$-apo-8'-carotenal, astacene and methyl bixin respectively.

\subsection{Adsorption kinetics}

The Roginsky-Zeldovich equation for adsorption kinetics in a modiffed form is given by (Mallik et al 1979b)

$$
d m / d t=A \exp (-\beta m / k T),
$$

where $m$ is the amount of vapour adsorbed at time $t, A$ and $\beta$ are constants at a particular vapour pressure, $k$ is the Boltzmann constant and $T$ is the absolute temperature. The relation between $\sigma_{A}, \alpha, \beta$ and $t$ is given by

$$
\log \sigma_{A}=\frac{a k T}{\beta} \log \left(t+t_{0}\right)+\text { constant. }
$$

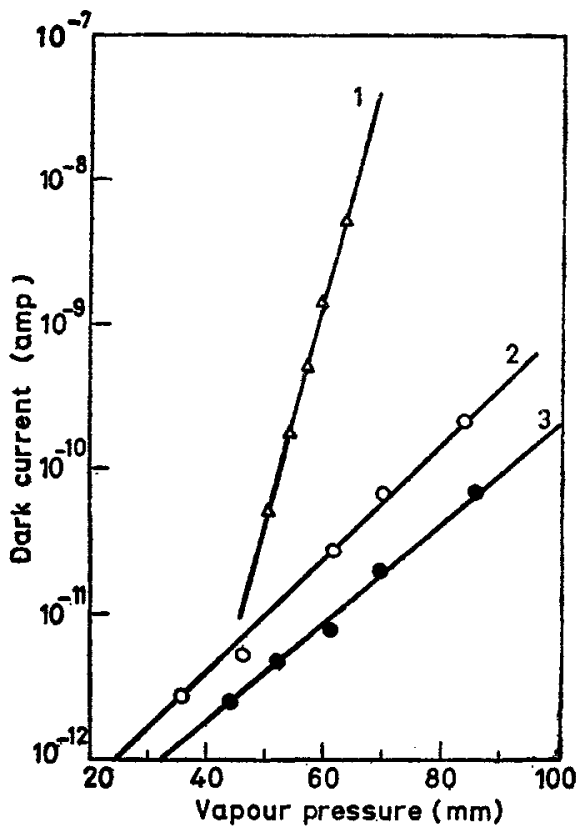

Figure 3. Change in the dark current $\left[I_{A}\left(m_{0}\right)\right]$ of $\beta$-apo-8'-carotenal, astacene and methyl bixin powder cells kept at $25^{\circ} \mathrm{C}$ as a function of the vapour pressure of ethyl acetate. The lines 1,2 and 3 correspond to $\beta$-apo-8'-carotenal, astacene and methyl bixin respectively. 
Thus, from any empirically chosen $t_{0}$, a linear plot of $\log \sigma_{A}$ versus $\log \left(t+t_{0}\right)$ is suggestod. With correctly chosen $t_{0}$, the plot of $m$ as a function of $\log \left(t+t_{0}\right)$ should give a straight line (Taylor and Thon 1952). Such plots for $\beta$-apo-8'-carotenal are shown in figure 4. The time indicated in the abscissa is the time measured from the initiation of adsorption. As in vitamin A (alcohol and acetate); in the initial region different slopes observed at different vapour pressu res show the vapour pressure-dependence of $\beta$. The higher the partial vapour pressure, the larger is the slope. We have estimated the values of $\beta / \alpha\left(=\beta^{\prime}\right)$ from the slopes to see its pressure dependence. In table 2 variation of $\beta^{\prime}$ with vapour pressure for ethyl acetate vapour adsorption on various polyenes is shown.

\subsection{Desorption kinetics}

Equation (2) is valid for the rate of adsorption. Since desorption is a reverse process of adsorption, equation for the rate of desorption can be written in a similar form with a positive sign in the exponent, i.e., by

$$
-d m / d t=A^{*} \exp \left(\beta^{*} m / k T\right) .
$$

Here, $\beta^{*} m$ is the activation energy for desorption. In the measurement of desorption, the experimental conditions are so arranged that there is no re-adsorption. The plots of logarithm of current versus logarithm of time for desorption of ethyl

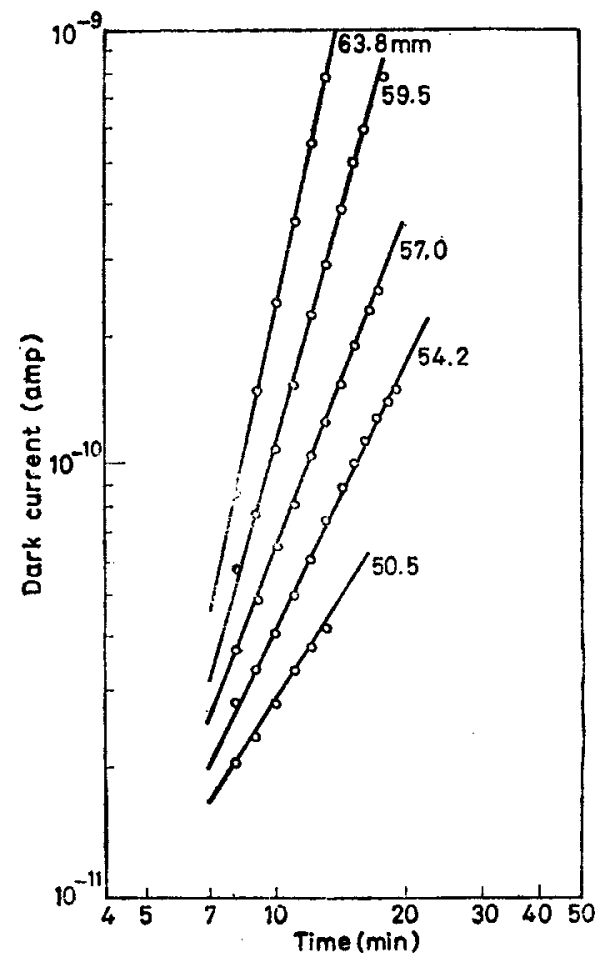

Figure 4. Adsorption kinetics data plotted according to modified Roginsky-Zeldovich equation for $\beta$-apo-8'-carotenal, 
Table 2. Vapour pressure dependence of the factors $\beta^{\prime}$ and $\beta^{* \prime}$ for vapour adsorption and desorption kinetics respectively in case of ethyl acetate vapour adsorption.

\begin{tabular}{|c|c|c|c|}
\hline Polyenes & $\begin{array}{c}\text { Vapour } \\
\text { pressure (mm) }\end{array}$ & $(\mathrm{eV})\left(\stackrel{\beta}{\times}^{\prime} 10^{-2}\right)$ & $(\mathrm{eV})\left(\begin{array}{l}\beta^{* \prime} \\
\left.\times 10^{-2}\right)\end{array}\right.$ \\
\hline \multirow{5}{*}{$\beta$-apo-8'-carotenal } & $50 \cdot 5$ & $1 \cdot 629$ & $8 \cdot 621$ \\
\hline & $54 \cdot 2$ & $1 \cdot 245$ & 1.919 \\
\hline & $57 \cdot 0$ & 0.994 & $1 \cdot 322$ \\
\hline & $59 \cdot 5$ & $0 \cdot 718$ & 1.015 \\
\hline & $63 \cdot 8$ & 0.555 & 0.808 \\
\hline \multirow{5}{*}{ Astacene } & $35 \cdot 8$ & 3.973 & $6 \cdot 733$ \\
\hline & $46 \cdot 0$ & $2 \cdot 568$ & $4 \cdot 591$ \\
\hline & $61 \cdot 0$ & $1 \cdot 422$ & $1 \cdot 720$ \\
\hline & $70 \cdot 0$ & $1 \cdot 168$ & $1 \cdot 250$ \\
\hline & $84 \cdot 0$ & 0.931 & 0.969 \\
\hline \multirow{5}{*}{ Methyl bixin } & $44 \cdot 0$ & $5 \cdot 714$ & $8 \cdot 312$ \\
\hline & $52 \cdot 0$ & $4 \cdot 131$ & 5.971 \\
\hline & $61 \cdot 0$ & $2 \cdot 526$ & $4 \cdot 322$ \\
\hline & $70 \cdot 0$ & 1.638 & $2 \cdot 215$ \\
\hline & $86 \cdot 0$ & $1 \cdot 039$ & $1 \cdot 431$ \\
\hline \multirow{5}{*}{ Vitamin A alcohol } & $31 \cdot 0$ & $1 \cdot 202$ & $2 \cdot 304$ \\
\hline & $35 \cdot 0$ & $1 \cdot 005$ & $1 \cdot 347$ \\
\hline & $37 \cdot 5$ & 0.930 & $1 \cdot 492$ \\
\hline & $43 \cdot 5$ & 0.759 & $1 \cdot 201$ \\
\hline & $50 \cdot 5$ & 0.543 & 0.798 \\
\hline \multirow{5}{*}{ Vitamin A acetate } & $31 \cdot 0$ & 0.819 & $1 \cdot 431$ \\
\hline & $35 \cdot 7$ & 0.753 & $1 \cdot 151$ \\
\hline & $41 \cdot 7$ & 0.660 & $1 \cdot 023$ \\
\hline & $48 \cdot 2$ & 0.601 & $0 \cdot 802$ \\
\hline & $55 \cdot 2$ & $0 \cdot 518$ & 0.648 \\
\hline
\end{tabular}

acetate vapour for $\beta$-apo- $8^{\prime}$-carotenal crystallites are shown in figure 5 . The time indicated in the abscissa is the time measured from the initiation of desorption. Similar plots have been made for other polyenes also. In table 2 we present the experimental values of $\beta^{* \prime}\left(=\beta^{*} / \alpha\right)$ obtained from these plots for ethyl acetate vapour desorption from different polyene crystallites. We have also included the date of vitamin A (alcohol and acetate) to show the generality of the kinetics of vapour adsorption and desorption in all these polyenes. As in $\beta^{\prime}, \beta^{* \prime}$ also decreases with increasing vapour pressure. For any particular pressure of ambient vapour, $\beta^{*}$ is larger than $\beta$.

The Roginsky-Zeldovich equation can be treated in different ways (Stone 1955), A simple two-stage process after Eley and Leslie (1964) as shown in figure 6 seems quite satisfactory to account for the experimental observations. In the first stage a mobile van der Waals' adsorption on the crystal surface gives a Lennard-Jones potential energy curve which is assumed to depend on the fraction of surface coverage. This stage is followed by a rate-determining transition over a potential energy 


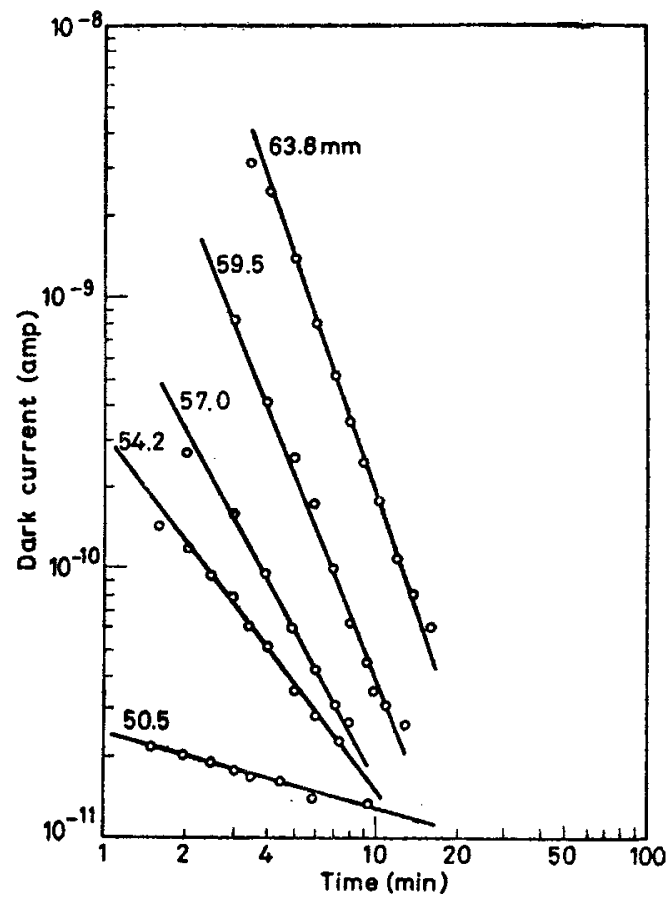

Figure 5. Desorption kinetics data plotted according to modified Roginsky-Zeldovich equation for $\beta$-apo-8'-carotenal.

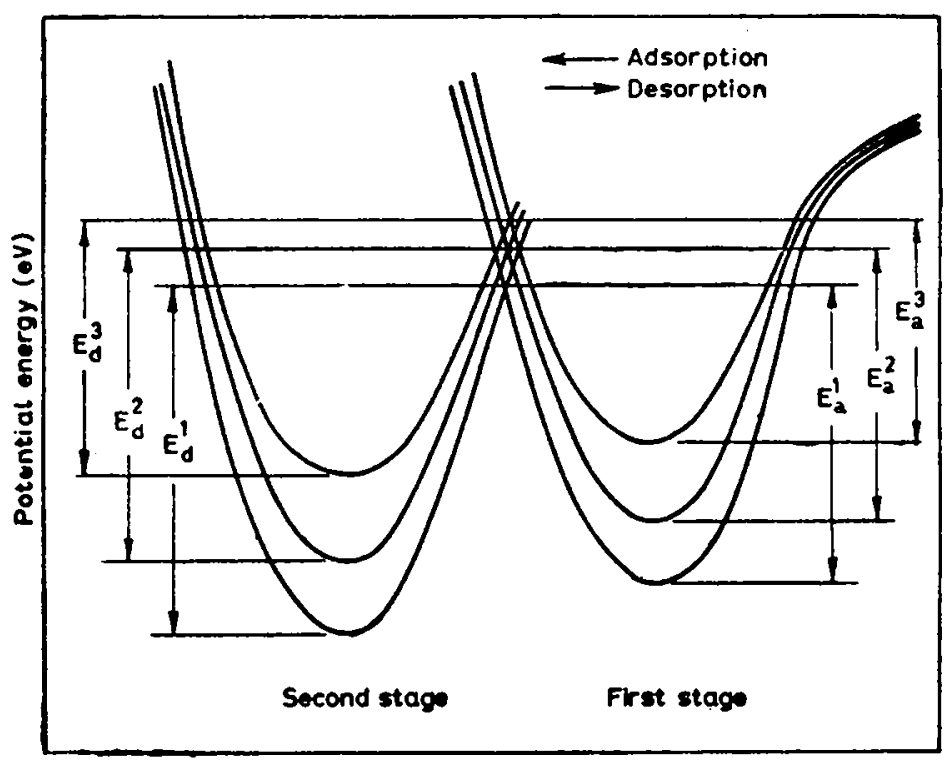

Reaction path

Figure 6. Potential energy curves for vapour adsorption in two stages in polyenes, explaining modified Roginsky-Zeldovich plots. $E_{d}^{1}, E_{2}^{2}$ and $E_{d}^{3}$ are activation energies of desorption in order of increasing pressure. Similarly $E_{\text {, }}$ 's are activation exergies for adsorption. 
barrier to the final stage of adsorption forming weakly bound complexes between the vapour molecules and the polyene crystallites. The barrier is formed by the intersection of the two potential curves. As more vapour molecules get physically adsorbed (van der Waals), a repulsive interaction between the dipoles will raise the potential energy curve for the first stage thereby lowering the barrier height giving decreasing activation energy of adsorption with increasing vapour pressure. As the surface coverage of the second stage rises, this potential curve also rises resulting in lowering of desorption activation energy with increasing pressure. The rise of the second stage curve is such that the minimum of this curve is always at lower energy than the minimum of the first stage, the energy difference between these two minima decreasing with increasing vapour pressure.

Here $\beta^{*} m$, the activation energy for desorption should, apart from a small entropy factor, be equal to the vapour-surface molecular complex. In table 2, we have shown the experimental value of $\beta^{* \prime}$. Unfortunately neither our experiments give any numerical value of $\alpha$, nor we have been able to measure $m$, the amount of vapour adsorbed. Any reliable estimate of the binding energy of various vapours with different polyenes has, therefore, not been possible. In the hydration of proteins, Rosenberg (1964) estimated $a \approx 2 \cdot 6$. For a similar value of $a$ for these cases of vapour desorption from polyene crystallites, the value of binding energy is $\approx 10^{-2} \mathrm{eV}$ per unit of $m$ desorbed, This low value of the binding energy accounts for easy and efficient desorption of these vapours from the polyene crystals.

\section{Ackmowledgements}

Thanks are due to the Council of Scientiftc and Industrial Research, New Delhi, for a fellowship to BM and to Prof. G S Kastha for his kind interest. Thanks are also due to $\mathrm{M} / \mathrm{s}$. Hoffman La-Roche, Switzerland, for a kind gift of the polyenes.

\section{References}

Eley D D and Leslie R B 1964 Advances in chemical physics (New York : Interscience) 7238 Misra T N, Rosenberg B and Switzer R 1968 J. Chem. Phys. 482096

Mallik B, Ghosh A and Misra T N 1979a Bull. Chem. Soc. Jpn. 522091

Mallik B, Ghosh A and Misra T N 1979b Proc. Indian Acad. Sci. (Chem. Sci.) A88 25

Mallik B, Ghosh A and Misra T N 1980 Jpn. J. Appl. Phys. 19207

Rosenberg B, Misra T N and Switzer R 1968 Nature (London) 217423

Rosenberg B 1964 Physical processes in radiation biology eds. L Augenstein, R Mason and

B Rosenberg (New York and London: Academic Press) p. 111

Taylor H A and Thon N 1952 J. Am. Chem. Soc. 744169 